\title{
Effects of Pinch Technique, Torque Direction and Sensation on the Pinch Force in Loaded and Unloaded Conditions
}

\author{
Poh Kiat $\mathrm{Ng}^{1}$, Adi Saptari ${ }^{2} \&$ Chiew Yean $\mathrm{Ng}^{3}$ \\ ${ }^{1}$ Faculty of Engineering and Technology, Multimedia University, Malacca, Malaysia \\ ${ }^{2}$ Faculty of Manucaturing Engineering, Technical University of Malaysia, Malacca, Malaysia \\ ${ }^{3}$ Department of Radiology, Columbia Asia Hospital, Puchong, Selangor, Malaysia \\ Correspondence: Poh Kiat Ng, Faculty of Engineering and Technology, Multimedia University, Jalan Ayer \\ Keroh Lama, Bukit Beruang, 75450 Malacca, Malaysia. E-mail: pkng@mmu.edu.my
}

Received: September 3, 2015

Accepted: September 19, 2015

Online Published: November 30, 2015

doi:10.5539/mas.v9n13p164

URL: http://dx.doi.org/10.5539/mas.v9n13p164

The research is financed by the Ministry of Education Malaysia through the Fundamental Research Grant Scheme (Ref No.: MMU/RMC-PL/AL/FRGS/2013/006).

\begin{abstract}
Many daily hand-related tasks require the ability to produce and control pinch forces to handle small objects. While there have been studies of various parameter effects on grip force, there appear to be no studies that investigate these effects on pinch force. This study aims to determine the effects of pinch technique, torque direction and sensation on pinch force with an emphasis on screw knobs under loaded and unloaded conditions. A total of 30 manual workers participated in the study. The workers were required to operate loaded and unloaded screw knobs in clockwise and counterclockwise directions to produce pinch forces using reduced and increased sensations and 3 types of common pinch techniques. The data was analysed using the analysis of variance via Minitab 16. Results for both conditions showed that pinch force is significantly affected by torque direction, sensation and pinch technique. However, the interaction effects of sensation and torque direction on pinch force appeared to be insignificant for both conditions. This is because the effects of increased sensation which reduce pinch force were neutralised by the effects of clockwise torque direction which increase force, while the effects of reduced sensation which increase pinch force were compensated by the effects of counterclockwise rotations which reduce pinch force. This study serves as a fundamental guideline for researchers and designers to improve hand tool designs operated with pinch grips so that they are safer and more ergonomic for manual tasks in the industry.
\end{abstract}

Keywords: pinch technique, torque direction, sensation, pinch force, hand-related musculoskeletal disorders, hand injuries, load condition

\section{Introduction}

Hands and fingers are underlying tools for high precision manufacturing work and are often used to pinch, grip and manipulate objects such as fasteners, clips and electronic components. However, awkwardly positioned, high-force pinch grip exertions can perhaps lead to hand-related injuries and cumulative trauma disorders (Ellis, Bloswick, Sesek, Mann, Thiese \& Hegman, 2004).

In Malaysia, it was reported that out of 428 industrial accidents, $24.7 \%$ of them included occupational hand injuries, with over $46.2 \%$ them considered as severe hand injuries (Al-Husuny, 2011). Besides that, hand-related musculoskeletal pain was found to be prevalent among Malaysian manual workers, with over $48 \%$ of them experiencing pain in the hands during manual tasks (Nur, Dawal \& Dahari, 2014).

In association to the preceding evidence, studies found that the type of pinch technique significantly affects grip force (Enders \& Seo, 2011) and finger-tip pinch techniques can lead to chronic pain in the wrist and digits (Ellis et al., 2004). Researchers posit that grip force is higher when an object is gripped and turned in a clockwise direction compared to a counterclockwise direction (Seo \& Armstrong, 2006; Seo \& Armstrong, 2008; Seo, Armstrong, Ashton-Miller \& Chaffin, 2007; Seo, Armstrong, Chaffin \& Ashton-Miller, 2008a; Seo, Armstrong, 


\section{Chaffin \& Ashton-Miller, 2008b).}

There have been some studies on how increased tactile sensation reduces grip force and how reduced tactile sensation increases grip force (Enders \& Seo, 2011; Huysmans, Hoozemans, Visser \& Van-Dieen, 2008). It was also found that grip force increases for gripped objects with loads over 3 times more than those without loads (Winstein, Abbs \& Petashnick, 1991).

While there have been studies conducted on grip force, there appear to be no studies that investigate the effects of pinch technique, torque direction and sensation on pinch force. Hence, the aim of this study is to determine the effects of these parameters on pinch force, with an emphasis on loaded and unloaded conditions.

Pinch grips involve the gripping of an object between the pads of the thumb and other fingers (Kumar, 2008; Smith \& Benge, 1985). Pinch force refers to the measure of actual distributed force on fingers in a pinch grip (Freund, Toivonen \& Takala, 2002). Most daily hand-related tasks require the ability to produce and control pinch force to handle small objects (Iyengar, Santos, Ko \& Aruin, 2009; Rost, Nowak, Timmann \& Hermsdorfer, 2005). Pinch force is also applied to produce thrust forces and torque to prevent objects from rotating and sliding away from fingers (Seo \& Armstrong, 2008).

A pinch technique is an orderly procedure of gripping with a thumb and finger. The commonly used pinch techniques in manufacturing and daily living activities include the 3-jaw chuck pinch, lateral pinch and pulp-2 pinch (Ellis et al., 2004; Shih \& Ou, 2005; Valero-Cuevas, Smaby, Venkadesan, Peterson \& Wright, 2003). A 3-jaw chuck pinch is performed by placing the object between the distal phalange's fingertips and thumb pad (Ellis et al., 2004). A lateral pinch uses the thumb pad and the radial side of the index finger's middle phalange (Dempsey \& Ayoub, 1996; Smaby, Johanson, Baker, Kenney, Murray \& Hentz, 2004; Trew \& Everett, 2005). A pulp-2 pinch is executed using the thumb pad and the fore finger's pad (Dempsey \& Ayoub, 1996).

Hand torques can be applied in two directions namely clockwise and counterclockwise torque directions. Clockwise torque direction is defined as the twisting movement of the hand towards the inner forearm direction, whereas the counterclockwise torque direction is defined as the twisting movement of the hand towards the outer forearm direction (Seo et al., 2008a; Seo et al., 2008b). Seo et al. (2007) suggested that in a twisting activity, clockwise torque direction allows the exertion of greater grip force than counterclockwise torque direction.

Sensation is an occurrence when a stimulus or something that causes a physical or mental response, is received by one of the various sensory organs of the body and is fed into the brain (Tanaka \& Numazawa, 2004). As an active sensory organ, the hand can detect objects even when there is no visual input and can perceive different object shapes, object sizes and surfaces (Trew \& Everett, 2005).

Sensory-disabled individuals however, are diminished of their sense of touch due to sensory deficits of the median nerve, and they often face problems pertaining to manual motor activities (Moberg, 1962; Moberg, 1964; Moberg, 1975; Nowak, Hermsdorfer, Marquardt \& Topka, 2003). Their disability to use the hand in manipulative tasks causes them to frequently drop objects, easily crush fragile objects and normally face problems in buttoning their shirt while dressing (Nowak et al., 2003).

\section{Method}

In this study, the dependent variable is pinch force while the independent variables are pinch technique, torque direction and sensation. Three flexiforce sensors (from Tekscan ${ }^{\circledR}$ Inc) are used in this study to record pinch force. In order to simulate the feeling of reduced and increased sensation, cotton gloves (reduced sensation) and nitrile gloves (increased sensation) are used.

A total of 30 manual workers from a furniture manufacturing firm in Malaysia participated in this study. Prior to the experiment, these workers are asked to fill up a consent form to confirm their participation. The 3 flexiforce sensors are attached to the participants' thumb, fore finger and middle finger. The nitrile and cotton gloves are cut into small pieces and attached on the tip of the sensors. A customised glove that exposed the thumb, fore finger and middle finger of the participants is used to allow the sensors to be firmly in position with the fingers and stagnant on the palm at all times. Figure 1 shows an illustration of how the sensors are attached according to the aforementioned procedures. 


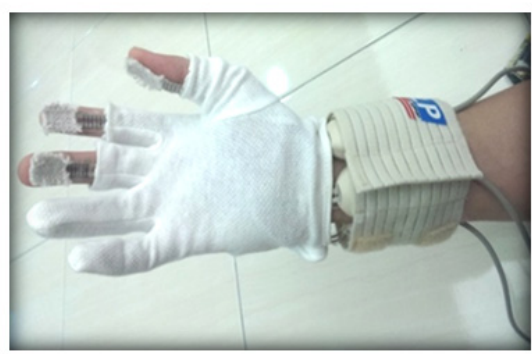

Figure 1. Attachment of Sensors to Fingers

Screw knobs are used as the test apparatus since many pinch-related activities involve screw knobs which are generally used in almost any machine, hand tool, device or furniture (Monroe, 2013). The knobs are to be mounted on wooden structures without any loading (unloaded) as shown in Figure 2. The overall structure originated from the design and development work of previous researchers $(\mathrm{Ng}$, Boon, Chai, Leh, Bee \& Saptari, 2013; Ng, Chai, Leh, Bee, Boon \& Saptari, 2013; Ng, Jee, Saptari \& Leau, 2013; Ng, Leh, Bee, Boon, Chai \& Jee, 2013), who only managed to conduct a psychophysical test with the apparatus.

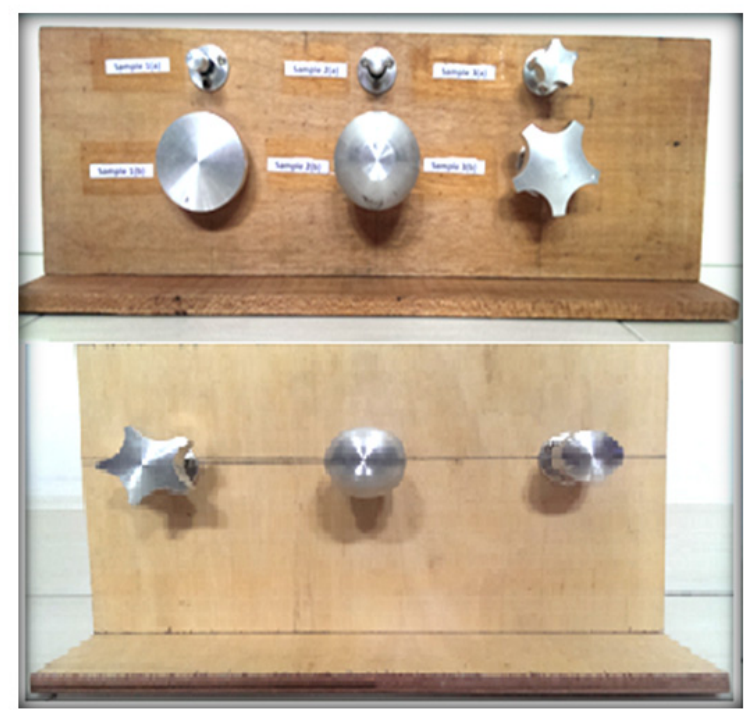

Figure 2. Wooden Structures without Loading (Unloaded)

Another wooden structure is also developed in order to simulate the presence of some loading effect (see Figure 3). Actual door knob mechanisms are embedded into this loaded structure. The frontend of the door knobs are modified to an adapter which allowed the screw knobs to be attached. This basically allowed the screw knobs to be twisted with some form of loading effect. When the screw knobs are fitted into the holes of the adapter and turned, the door knob mechanism within the adapter creates a counter force that simulates the presence of this loading effect. 


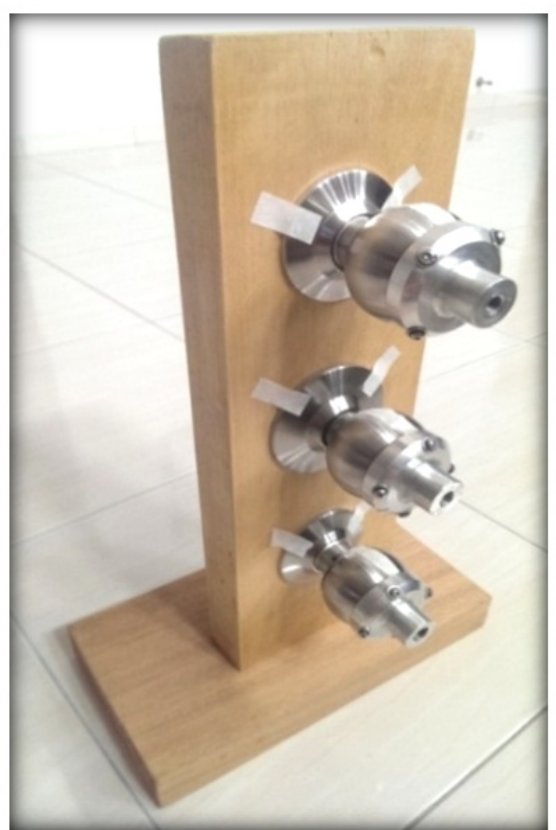

Figure 3. Wooden Structure with Loading (Loaded)

\section{Experimental Protocol}

Each participant is required to pinch the screw knobs with the 3 commonly used pinch techniques, as identified by Smith \& Benge (1985). The knobs are to be twisted using both clockwise and counterclockwise torque directions.

Figure 4 (a) presents an example of how a 3-jaw chuck pinch technique is applied, while Figure 4 (b) shows how a pulp-2 pinch technique is applied. Figure 4 (c) shows an example of how a lateral pinch technique is applied. The recorded pinch forces are then analysed using the analysis of variance (ANOVA) to determine the significance of the main and interaction effects of pinch technique, torque direction and sensation on pinch force.

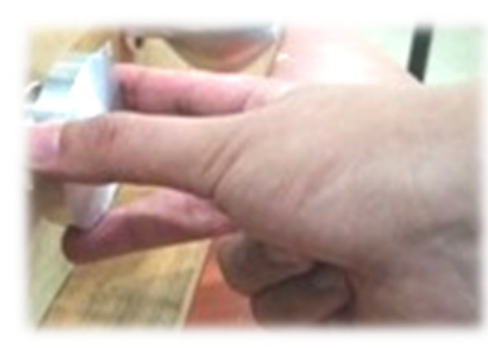

(a)

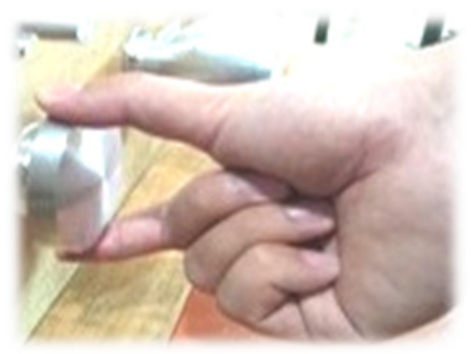

(b)

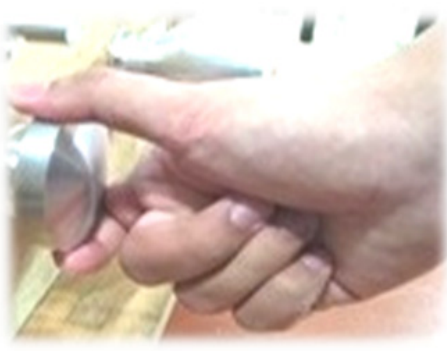

(c)

Figure 4. The 3 Commonly Used Pinch Techniques (Smith \& Benge, 1985)

\section{Results}

Table 1 shows the ANOVA results on the significances of the main and combinatorial effects on pinch force. Based on the results for the loaded and unloaded condition, it appears that pinch force is significantly affected by all the main factors (torque direction, sensation and pinch technique) $(p<0.05)$. Pinch force is also significantly affected by two out of three interactions (sensation $\times$ pinch technique, pinch technique $\times$ torque direction) $(p<$ 0.05). However, the interaction effects of sensation and torque direction on pinch force appear to be not significant for both the loaded and unloaded conditions $(p>0.05)$. 
Table 1. Significances of the Main Effects on Pinch Force

\begin{tabular}{lcccc}
\hline \multirow{2}{*}{ Factors } & \multicolumn{2}{c}{ Loaded } & \multicolumn{2}{c}{ Unloaded } \\
& $\boldsymbol{p}$ & Significance & $\boldsymbol{p}$ & Significance \\
\hline Torque direction & 0.000 & Significant & 0.000 & Significant \\
Sensation & 0.000 & Significant & 0.000 & Significant \\
Pinch technique & 0.000 & Significant & 0.000 & Significant \\
Sensation $\times$ pinch technique & 0.000 & Significant & 0.000 & Significant \\
Sensation $\times$ torque direction & 0.495 & Not Significant & 0.356 & Not Significant \\
Pinch technique $\times$ torque direction & 0.018 & Significant & 0.028 & Significant \\
\hline
\end{tabular}

\subsection{Sensation and Pinch Force}

Figure 5 (a) and Figure 5 (b) show the factorial plot results for the effects of tactile sensation on pinch force for the loaded and unloaded conditions. The results of the loaded condition in Figure 5 (a) suggest that the mean pinch force exerted with reduced sensation is $82.3267 \mathrm{~g}$ (highest), which decreases by about $9.85 \%$ with the application of increased sensation to a mean pinch force of $74.2158 \mathrm{~g}$ (lowest). The results of the unloaded condition in Figure 5 (b) similarly suggest that the mean pinch force exerted with reduced sensation is $81.7836 \mathrm{~g}$ (highest), which decreases by about $9.81 \%$ with the application of increased sensation to a mean pinch force of $73.7593 \mathrm{~g}$ (lowest). The force patterns for both of these results (loaded and unloaded conditions) are observed to be similar to each other.

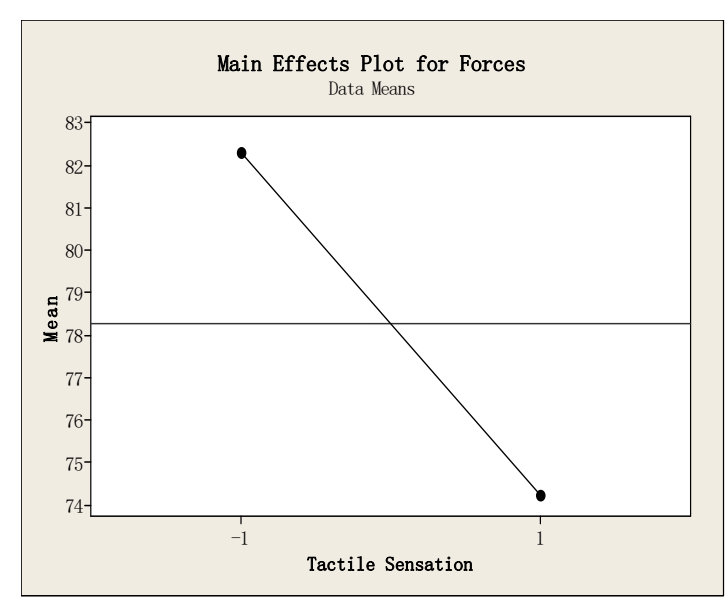

(a) Loaded

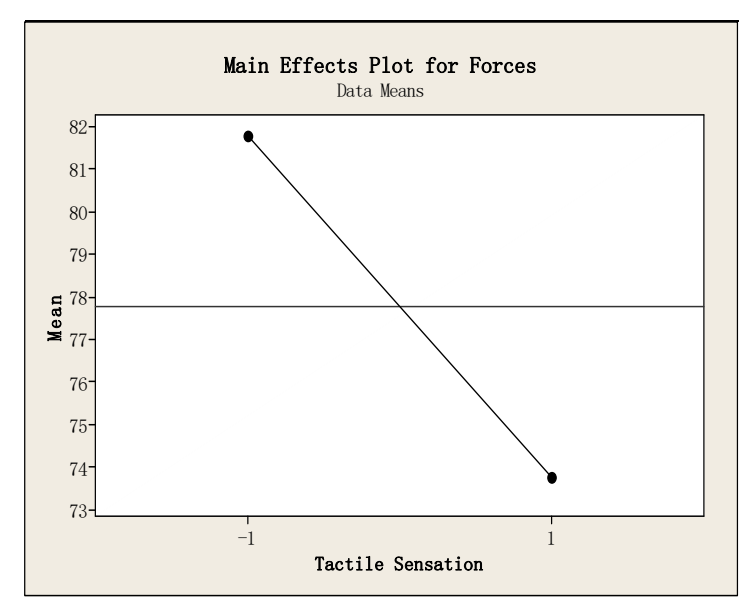

(b) Unloaded

Figure 5. Factorial Plot of Tactile Sensation

(Note: -1 refers to reduced sensation, 1 refers to increased sensation)

\subsection{Pinch Technique and Pinch Force}

Figure 6 (a) and Figure 6 (b) show the factorial plot results for the effects of pinch technique on pinch force for the loaded and unloaded conditions. The results of the loaded condition in Figure 6 (a) suggest that the mean pinch force exerted by the lateral pinch (0) is $85.6251 \mathrm{~g}$ (highest), which is $6.63 \%$ higher than the force exerted by the pulp-2 pinch (-1) (79.9518g) (medium) and 19.14\% higher than the force exerted by the 3 -jaw chuck (1) (69.2368g) (lowest). These results are similar to those of the unloaded condition in Figure 6 (b), where the mean pinch force exerted by the lateral pinch (0) is $84.9555 \mathrm{~g}$ (highest), which is $6.60 \%$ higher than the force exerted by the pulp-2 pinch (-1) (79.3526g) (medium) and 18.77\% higher than the force exerted by the 3 -jaw chuck (1) (69.0063g) (lowest). 


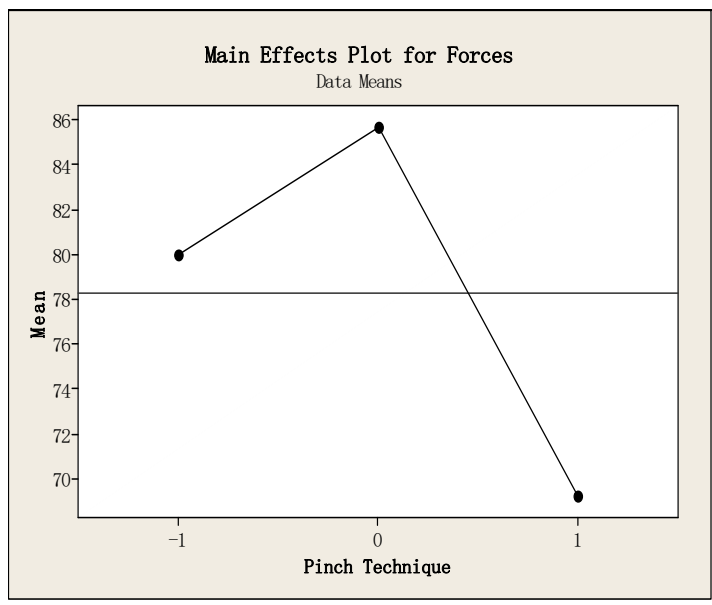

(a) Loaded

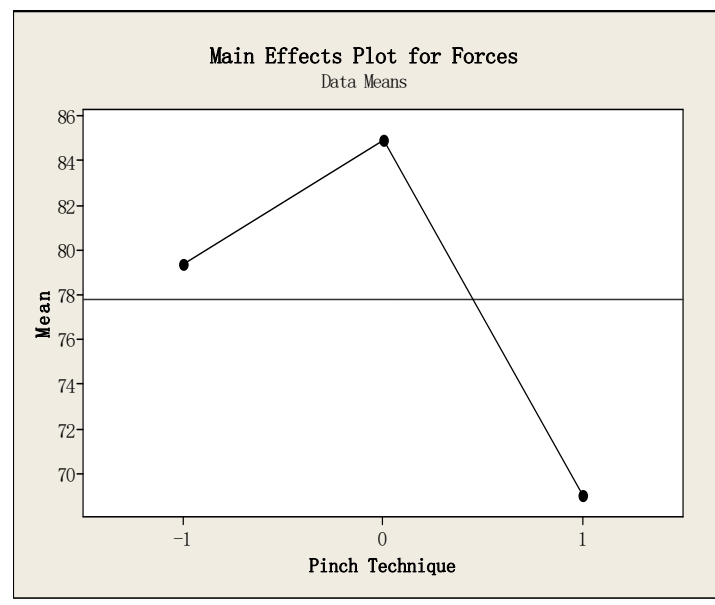

(b) Unloaded

Figure 6. Factorial Plot of Pinch Technique

(Note: -1 refers to pulp-2 pinch, 0 refers to lateral pinch, 1 refers to 3 -jaw chuck pinch)

\subsection{Torque Direction and Pinch Force}

Figure 7 (a) and Figure 7 (b) show factorial plot results for the effects of torque direction on pinch force for the loaded and unloaded conditions. For the loaded condition in Figure 7 (a), the force exerted in a clockwise motion $(-1)$ appears to be higher than force exerted in a counterclockwise motion (1). The mean pinch force exerted with the clockwise torque direction (-1) is $79.6369 \mathrm{~g}$, which decreases by about $3.43 \%$ with the application of the counterclockwise direction (1) to a mean pinch force of $76.9056 \mathrm{~g}$. This result is similar for the unloaded condition.

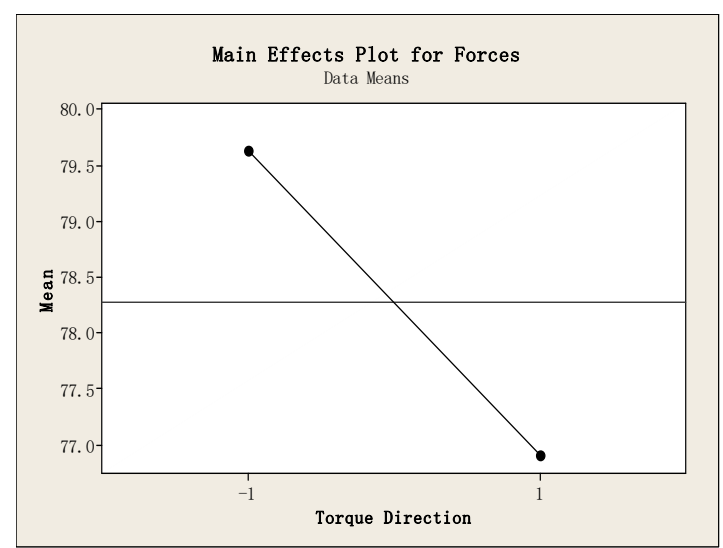

(a) Loaded

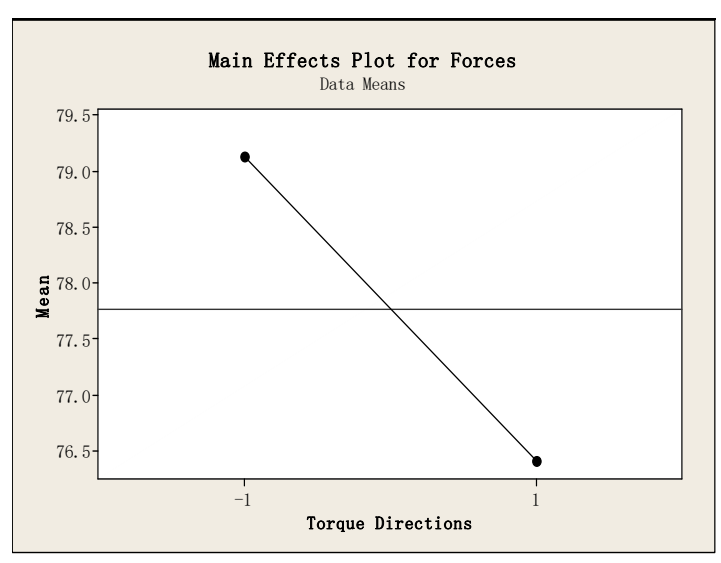

(b) Unloaded

Figure 7. Factorial Plot of Torque Direction

(Note: -1 refers to clockwise torque direction, 1 refers to counterclockwise torque direction)

\subsection{Sensation, Pinch Technique and Pinch Force}

Figure 8 (a) and Figure 8 (b) show the interaction plot results for the effects of sensation and pinch technique on pinch force for the loaded and unloaded conditions. Both results show similar force patterns and confirm that pinch force is significantly affected by sensation and pinch technique $(p<0.05)$. It can be generally seen that reduced sensation (-1) allows participants to generate higher pinch forces compared to increased sensation (1).

It also appears that the effects of pinch technique on pinch force are more dominant than the effects of sensation since the pinch force patterns of the two figures look similar to those of Figure 6 (Factorial plot of pinch technique). If Figure 8 was cross-checked with Figure 6, a similarity can be seen in the pinch force pattern, where the lateral pinch generates the highest pinch force, followed by pulp-2 pinch and the 3 -jaw chuck pinch. 


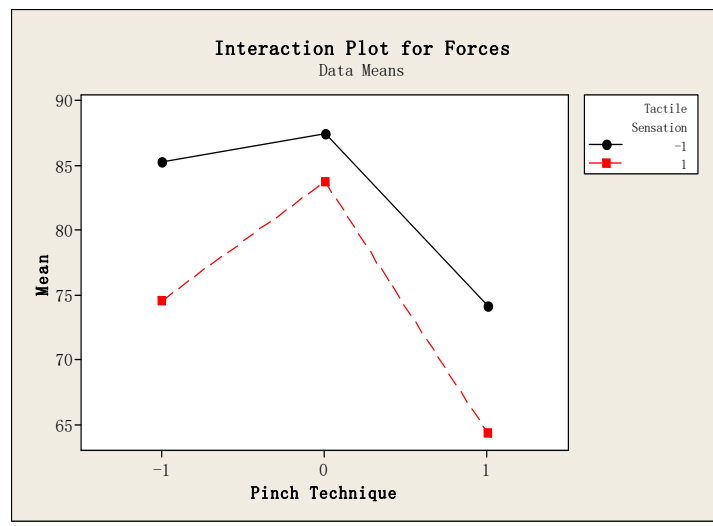

(a) Loaded

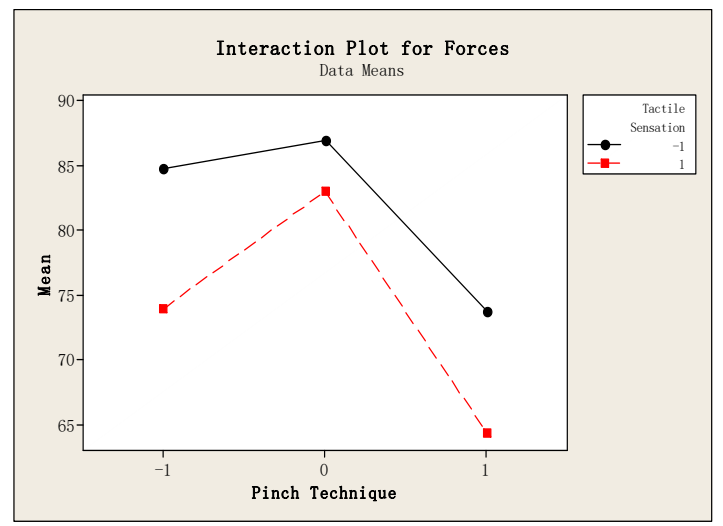

(b) Unloaded

Figure 8. Interaction Plot of Sensation and Pinch Technique

(Note for pinch technique: -1 refers to pulp-2 pinch, 0 refers to lateral pinch, 1 refers to 3 -jaw chuck pinch)

(Note for tactile sensation: -1 refers to reduced sensation, 1 refers to increased sensation)

In Figure 8 (a), the pulp-2 pinch force with reduced sensation (-1) is $85.3323 \mathrm{~g}$, which is $12.61 \%$ higher than the pulp-2 pinch force with increased sensation (1) (74.5714g). This is similarly seen for the unloaded condition in Figure 8 (b).

\subsection{Sensation, Torque Direction and Pinch Force}

Figure 9 (a) and Figure 9 (b) show the interaction plot results for the effects of sensation and torque direction on pinch force for the loaded and unloaded conditions. Both figures have similar pinch force patterns. However, the effects of sensation and torque direction on pinch force are not significant $(p>0.05)$. From the figures, it appears that the graphs are parallel to each other across the two different categories (sensation and torque direction), showing that there are no significant changes in pinch forces when different sensations and torque directions are applied. Furthermore, when cross-checked against Figure 7 (Factorial plot for torque direction) and Figure 5 (Factorial plot for tactile sensation), it is found that the pattern of the forces are consistently similar.

For the loaded condition, the clockwise torque direction (-1) with reduced sensation (-1) generates a mean pinch of $83.9010 \mathrm{~g}$, which is $10.16 \%$ higher than the mean pinch force generated with increased sensation (1) $(75.3727 \mathrm{~g})$. The counterclockwise torque direction (1) with reduced sensation $(-1)$ generates a mean pinch force of $80.7523 \mathrm{~g}$, which is $9.53 \%$ higher than the mean pinch force generated with increased sensation (1) $(73.0590 \mathrm{~g})$. Similar results are seen for the unloaded condition.

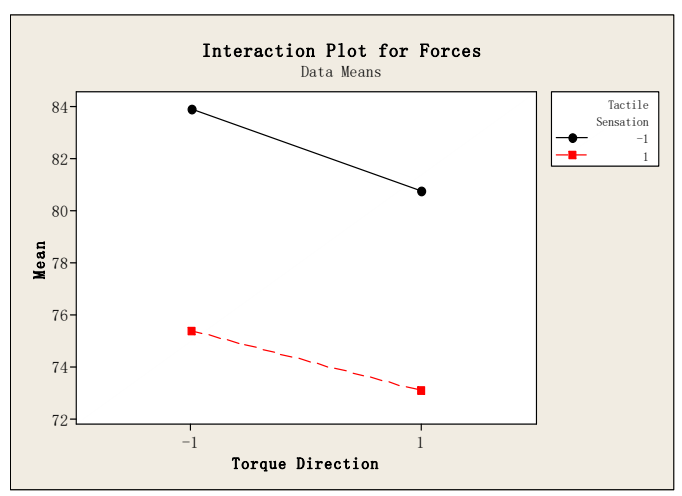

(a) Loaded

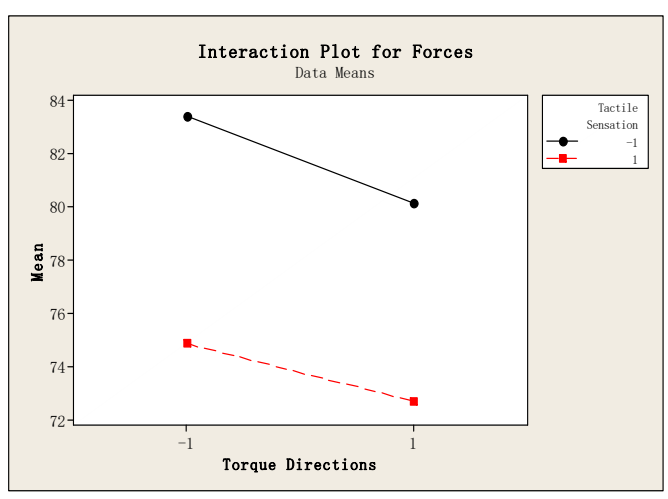

(b) Unloaded

Figure 9. Interaction Plot of Sensation and Torque Direction

(Note for torque direction: -1 refers to clockwise direction, 1 refers to counterclockwise direction)

(Note for tactile sensation: -1 refers to reduced sensation, 1 refers to increased sensation) 


\subsection{Pinch Technique, Torque Direction and Pinch Force}

Figure 10 (a) and Figure 10 (b) show the interaction plot results for the effects of pinch technique and torque direction on pinch force for the loaded and unloaded conditions. From the figures, it appears that the pinch force patterns across the 3 pinch techniques are different. In each figure, it appears that the graphs are not parallel to one another, indicating that a significant change in pinch forces occurs across varying pinch techniques and torque directions. This confirms that the interaction effects of sensation and object size on pinch force are significant $(p<0.05)$. It also appears that the effects of torque directions on pinch force are more dominant than the effects of pinch techniques since the pinch force patterns of the two figures look similar to those of Figure 8 (Factorial plot for torque direction).

When observing the force patterns based on pinch techniques, it appears that these factorial plots for both structures are consistent with the ones in Figure 6 (Factorial plot for pinch technique). Based on this cross-reference, the lateral pinch (0) generates the highest pinch force, followed by the pulp-2 pinch (-1) and 3-jaw chuck pinch (1).

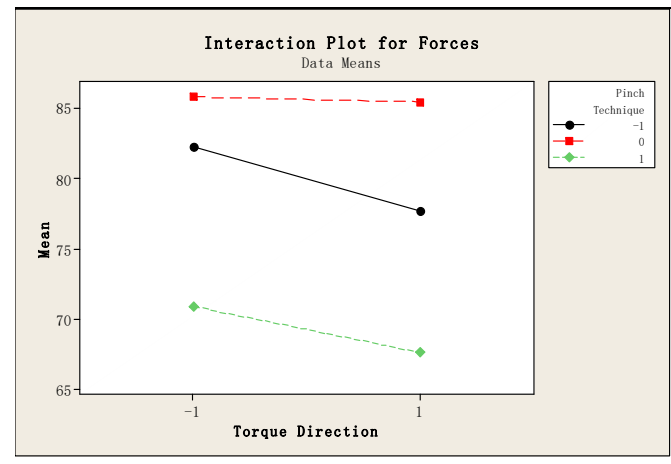

(a) Loaded

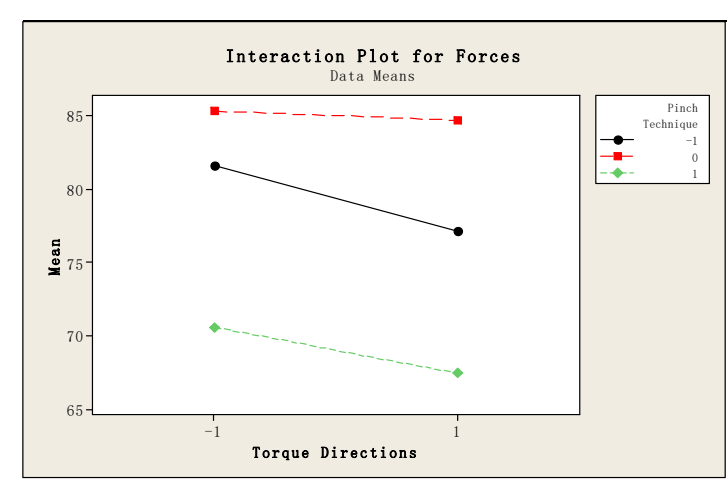

(b) Unloaded

Figure 10. Interaction Plot of Pinch Technique and Torque Direction

(Note for torque direction: -1 refers to clockwise direction, 1 refers to counterclockwise direction)

(Note for pinch techniques: -1 refers to pulp-2 pinch, 0 refers to lateral pinch, 1 refers to 3 -jaw chuck pinch)

\section{Discussion}

Effects of sensation on pinch force. This study proved that pinch force is significantly affected by sensation for the loaded and unloaded conditions $(p<0.05)$. The results show that the average pinch force exerted for reduced sensations is higher than the pinch force exerted for increased sensations. This suggests that participants tended to exert higher pinch forces on low-friction surfaces compared to high-friction surfaces. The results are consistent with the findings of other researchers who suggested that increased tactile sensation reduces normal force and reduced tactile sensation increases normal force (Enders \& Seo, 2011; Huysmans et al., 2008). Reduced sensation can decrease the acuity of tactile information obtained with the fingers and cause a reduction in friction between the fingers and the object, leading to a tendency to exert higher grip forces (Huysmans et al., 2008).

Effects of pinch technique on pinch force. Pinch force is significantly affected by pinch technique for both the loaded and unloaded conditions $(p<0.05)$. Findings also suggest that lateral pinch techniques allow individuals to generate the highest amount of pinch force, followed by the pulp-2 and 3-jaw chuck pinch technique.

Unlike other pinch techniques which utilise the pad of the fingertips, the lateral pinch uses the lateral aspect of the index finger. The volar and the lateral aspects in these pinch techniques may have different densities of papillary ridges and sweat glands, resulting in different coefficients of friction (Peaslee, 2007; Seo, 2009; Smith, Cadoret \& St-Amour, 1997; Zackrisson, Eriksson, Hosseini, Johnels \& Krogstad, 2008).

Since the surface of the index finger's lateral aspect is more slippery compared to the pad of finger, slippages tend to occur when lateral pinch is used. Due to this skin-object slippage, participants may have attempted to increase the force of their pinch grip voluntarily in order to completely turn the knobs. The results also show that the 3-jaw chuck pinch generates the lowest pinch force. The fact that the 3-jaw chuck pinch utilises three fingers 
to pinch perhaps allowed the participants to have better leverage and control while turning the knobs compared to the pulp-2 pinch. The improved control with three fingers may have loosened the muscle coordination requirement, which led to the reduction of pinch force exertion (since it did not take as much effort to pinch and turn the knobs as compared to the pulp-2 pinch).

Effects of torque direction on pinch force. Pinch force is significantly affected by torque direction $(p<0.05)$ for both the loaded and unloaded conditions. The results also show that the average pinch force exerted in a clockwise motion is higher than the average pinch force applied in a counterclockwise motion. Previous studies on hand grip force suggest that it is noticeable for greater torque to exist in clockwise torque directions than counterclockwise torque directions (Seo et al., 2007; Seo et al., 2008a). This is because the skin friction produced by twisting an object in a clockwise torque direction can flex the distal phalanges and increase the normal force and torque (Seo et al., 2007; Seo et al., 2008a; Seo et al., 2008b). These statements on grip force are consistent with the current findings on torque direction and pinch force.

Effects of sensation and pinch technique on pinch force. Pinch force is significantly affected by sensation and pinch technique for both the loaded and unloaded conditions $(p<0.05)$. Researchers suggested that pinch force can be different between lateral pinches and other types of finger tip pinches (Nakazawa, Ikeura \& Innoka, 2000; Seo, 2009). Participants may have increased the force of their pinch grip voluntarily due to the lack of friction when turning the knobs using the lateral pinch technique. In this study, it appears that the capacity of the lateral pinch technique in generating high forces is amplified with the employment of reduced sensation (Dempsey \& Ayoub, 1996; Fathallah, Kroemer \& Waldron, 1991; Huysmans et al., 2008), since this combination generated the highest pinch force.

Effects of sensation and torque direction on pinch force. The effects of sensation and torque direction on pinch force are not significant for both the loaded and unloaded conditions $(p>0.05)$. The results imply that the combination of sensation and torque direction does not spur significant changes in pinch force. This may be because the effects of increased tactile sensation which reduce normal force (Enders \& Seo, 2011; Huysmans et al., 2008) may have been neutralised by the effects of clockwise torque direction which increase the force (Seo, 2008; Seo et al., 2007; Seo et al., 2008b). In contrast, the effects of reduced sensation which cause a tendency of higher pinch force generation (Huysmans et al., 2008) may have been compensated by the effects of counterclockwise rotations which reduce pinch force due to the lack of available grip contact area (Seo et al., 2008b).

Effects of pinch technique and torque direction on pinch force. Pinch force is significantly affected by pinch technique and torque direction for both the loaded and unloaded conditions $(p<0.05)$. The rationales justified for this phenomenon are similar to those for Figure 7 due to similarities in the force patterns. However, there appears to be almost no difference in forces at all when the lateral pinch (0) is used with clockwise and counterclockwise torque directions (approximately $0.4 \%$ difference only). Hence, it is found that in a combinatorial study among pinch technique, torque direction and pinch force, the effects from the lateral pinch technique are more distinguishable than the effects from the different torque directions.

\section{Conclusion}

The main effects of pinch technique, torque direction and sensation on pinch force are found to be significant. This study presents several underlying observations:

- The average pinch force exerted in a clockwise motion is generally higher than the average pinch force applied in a counterclockwise motion because the skin friction produced by turning the object in a clockwise torque direction can flex the distal phalanges and increase the normal force.

- The average pinch force exerted for reduced sensation is higher than the pinch force exerted for increased sensation due to the reduced friction experienced during the pinch activity and the increased possibility of skin-object slippages, which caused participants to voluntarily increase their force exertion to avoid these slippages to happen.

- The lateral pinch technique also allows individuals to generate the highest pinch force, followed by the pulp-2 and 3-jaw chuck pinch technique. Similar to the concept of reduced sensation, since the surface of the lateral aspect of the index finger is more slippery compared to the pads of other fingers, participants may have attempted to increase the force of their lateral pinch voluntarily in order to completely turn the knobs without slipping.

- The combination of the lateral pinch technique and reduced sensation not only significantly affects pinch force, but also has the ability to generate the highest pinch force. It also appears that the effects of pinch techniques on pinch force are more dominant than the effects of sensations. 
- The effects of increased sensation which reduce pinch force are neutralised by the effects of clockwise torque direction which increase force, while the effects of reduced sensation which increase pinch force are compensated by the effects of counterclockwise rotations which reduce pinch force.

- The combination of pinch technique and torque direction significantly affects pinch force due to the difference in friction force direction across various pinch techniques and the different effects of the clockwise and counterclockwise torque directions. The effects of torque directions on pinch force are more dominant than the effects of pinch techniques.

This study enhances the knowledge of the effects of different pinch parameters on pinch force. With these findings, designers can potentially design superior ergonomic hand tools that are operated with pinch grips to be safer and more suitable for certain manual tasks in the industry.

For future directions of this research, it is proposed that the impacts of various muscular activities on pinch force are be investigated by using the SEMG (Surface Electromyography) which can record signals from the Thenar muscles during a pinch grip. The investigation of muscular contribution in pinch grips would be beneficial for the understanding of the pinch strength capability of individuals during a pinching activity. It also helps identify the underlying muscles responsible in generating a certain amount of pinch force. It may be important for researchers to consider the frequency of pinch grips and the ability of maintaining the pinch grip forces with time due to fatigue.

\section{Acknowledgements}

This research was supported by the Fundamental Research Grant Scheme (Ref No.: MMU/RMC-PL/AL/FRGS/2013/006) from the Malaysian Ministry of Education. The data presented, statements made and views expressed are solely the responsibility of the authors.

\section{References}

Al-Husuny, A. N. A. (2011). Assessment of Work-Related Hand Injuries at Hospital Serdang, Selangor, Malaysia. (Master of Science Dissertation), Universiti Putra Malaysia, Serdang, Malaysia.

Dempsey, P. G., \& Ayoub, M. M. (1996). The Influence of Gender, Grasp Type, Pinch Width and Wrist Position on Sustained Pinch Strength. International Journal of Industrial Ergonomics, 17(3), 259-273. http://dx.doi.org/10.1016/0169-8141(94)00108-1

Ellis, E. A., Bloswick, D. S., Sesek, R. F., Mann, C., Thiese, M. S., \& Hegman, K. T. (2004). The Effect of Pinch Grip on Upper Extremity Cumulative Trauma Disorders in Female Garment Workers. (Master of Science Dissertation), University of Utah, Salt Lake City, Utah, USA.

Enders, L. R., \& Seo, N. J. (2011). Phalanx Force Magnitude and Trajectory Deviation Increased During Power Grip with an Increased Coefficient of Friction at the Hand-Object Interface. Journal of Biomechanics, 44(8), 1447-1453. http://dx.doi.org/10.1016/j.jbiomech.2011.03.020

Fathallah, E. A., Kroemer, K. H. E., \& Waldron, R. L. (1991). A New Finger Strength (Pinch) Gauge. $\begin{array}{lllll}\text { International Journal of Industrial } & \text { Ergonmics, } & 7(1), & 71-72 .\end{array}$ http://dx.doi.org/10.1016/0169-8141(91)90060-Y

Freund, J., Toivonen, R., \& Takala, E. P. (2002). Grip Forces of the Fingertips. Clinical Biomechanics, 17(7), 515-520. http://dx.doi.org/10.1016/S0268-0033(02)00043-8

Huysmans, M. A., Hoozemans, M. J. M., Visser, B., \& Van-Dieen, J. H. (2008). Grip Force Control in Patients with Neck and Upper Extremity Pain and Healthy Controls. Clinical Neurophysiology, 119(8), 1840-1848. http://dx.doi.org/10.1016/j.clinph.2008.04.290

Iyengar, V., Santos, M. J., Ko, M., \& Aruin, A. S. (2009). Effect of Contralateral Finger Touch on Grip Force Control in Individuals with Multiple Sclerosis. Clinical Neurophysiology, 120(3), 626-631. http://dx.doi.org/10.1016/j.clinph.2008.12.029

Kumar, S. (2008). Biomechanics in Ergonomics (2nd ed.). Boca Raton: CRC Press.

Moberg, E. (1962). Criticism and Study of Methods for Examining Sensibility in the Hand. Neurology, 12(1), 8-19. http://dx.doi.org/10.1212/WNL.12.1.8

Moberg, E. (1964). Aspects of Sensation in Reconstructive Surgery of the Upper Extremity. Journal of Bone and Joint Surgery, 46(4), 817-825

Moberg, E. (1975). Hand Surgery and the Development of Hand Prostheses. Scandinavian Journal of Plastic and Reconstructive Surgery, 9(3), 227-230. http://dx.doi.org/10.3109/02844317509022870 
Monroe. (2013). Industrial Knobs. Retrieved April 9, from http://catalog.monroeengineering.com/category/knobs

Nakazawa, N. N., Ikeura, R., \& Innoka, H. (2000). Characteristics of Human Fingertips in the Shearing Direction. Biological Cybernetics, 82(3), 207-214. http://dx.doi.org/10.1007/s004220050020

Ng, P. K., Boon, Q. H., Chai, K. X., Leh, S. L., Bee, M. C., \& Saptari, A. (2013). The Roles of Shape and Size in the Pinch Effort of Screw Knobs. Paper presented at the 4th International Conference on Mechanical and Manufacturing Engineering, Bangi-Putrajaya, Malaysia. Abstract retrieved from http://bit.ly/1 WbrDCr

Ng, P. K., Chai, K. X., Leh, S. L., Bee, M. C., Boon, Q. H., \& Saptari, A. (2013). Applying Clockwise and Counterclockwise Torque Directions in Pinch Grips: A Descriptive Study. Paper presented at the 4th International Conference on Mechanical and Manufacturing Engineering, Bangi-Putrajaya, Malaysia. Abstract retrieved from http://bit.ly/1Wbrl8Z

Ng, P. K., Jee, K. S., Saptari, A., \& Leau, J. X. (2013). Familiarity with Office Equipment Reduces Human Errors and Accidents: A Survey. Paper presented at the International Conference on Advances in Mechanical and Manufacturing Engineering, Kuala Lumpur, Malaysia. Abstract retrieved from http://bit.ly/1KZasC9

Ng, P. K., Leh, S. L., Bee, M. C., Boon, Q. H., Chai, K. X., \& Jee, K. S. (2013). The Effects of Different Tactile Sensations on Pinch Effort. Paper presented at the 4th International Conference on Mechanical and Manufacturing Engineering, Bangi-Putrajaya, Malaysia. Abstract retrieved from http://bit.ly/1KrdVDX

Nowak, D. A., Hermsdorfer, J., Marquardt, C., \& Topka, H. (2003). Moving Objects with Clumsy Fingers: How Predictive Is Grip Force Control in Patients with Impaired Manual Sensibility? Clinical Neurophysiology, 114(3), 472-487. http://dx.doi.org/10.1016/S1388-2457(02)00386-3

Nur, N. M., Dawal, S. Z. M., \& Dahari, M. (2014). The Prevalence of Work Related Musculoskeletal Disorders among Workers Performing Industrial Repetitive Tasks in the Automotive Manufacturing Companies. Paper presented at the International Conference on Industrial Engineering and Operations Management, Bali, Indonesia. Abstract retrieved from http://iieom.org/ieom2014/pdfs/303.pdf

Peaslee, E. R. (2007). Human Histology: In Its Relations to Descriptive Anatomy, Physiology and Pathology (1st ed.). Whitefish, Montana: Kessinger Publishing.

Rost, K., Nowak, D. A., Timmann, D., \& Hermsdorfer, J. (2005). Preserved and Impaired Aspects of Predictive Grip Force Control in Cerebellar Patients. Clinical Neurophysiology, 116(6), 1405-1414. http://dx.doi.org/10.1016/j.clinph.2005.02.015

Seo, N. J. (2008). Biomechanical Models of Hand Coupling for Axial Torque and Push Exertions (1st ed.). Saarbrücken, Germany: VDM Verlag Dr. Mueller e.K.

Seo, N. J. (2009). Dependence of Safety Margins in Grip Force on Isometric Push Force Levels in Lateral Pinch. Ergonomics, 52(7), 840-847. http://dx.doi.org/10.1080/00140130802578555

Seo, N. J., \&Armstrong, T. J. (2006). The Effect of Torque Direction on Hand-Object Coupling. Paper presented at the Ameri can Society of Biomechanics 30th Meeting, Blacksburg, Virginia. Abstract retrieved from http://www.asbweb.org/conferences/2006/pdfs/88.pdf

Seo, N. J., \& Armstrong, T. J. (2008). Investigation of Grip Force, Normal Force, Contact Area, Hand Size and Handle Size for Cylindrical Handles. Human Factors: The Journal of the Human Factors and Ergonomics Society, 50(5), 734-744. http://dx.doi.org/10.1518/001872008X354192

Seo, N. J., Armstrong, T. J., Ashton-Miller, J. A., \& Chaffin, D. B. (2007). The Effect of Torque Direction and Cylindrical Handle Diameter on the Coupling between the Hand and a Cylindrical Handle. Journal of Biomechanics, 40(14), 3236-3243. http://dx.doi.org/10.1016/j.jbiomech.2007.04.023

Seo, N. J., Armstrong, T. J., Chaffin, D. B., \& Ashton-Miller, J. A. (2008a). The Effect of Handle Friction and Inward or Outward Torque on Maximum Axial Push Force. Human Factors: The Journal of the Human Factors and Ergonomics Society, 50(2), 227-236. http://dx.doi.org/10.1518/001872008X250692

Seo, N. J., Armstrong, T. J., Chaffin, D. B., \& Ashton-Miller, J. A. (2008b). Inward Torque and High-Friction Handles Can Reduce Required Muscle Efforts for Torque Generation. Human Factors: The Journal of the Human Factors and Ergonomics Society, 50(1), 37-48. http://dx.doi.org/10.1518/001872008X250610

Shih, Y. C., \& Ou, Y. C. (2005). Influences of Span and Wrist Posture on Peak Chuck Pinch Strength and Time Needed to Reach Peak Strength. International Journal of Industrial Ergonomics, 35(6), 527-536. 
http://dx.doi.org/10.1016/j.ergon.2004.12.002

Smaby, N., Johanson, M. E., Baker, B., Kenney, D. E., Murray, W. M., \& Hentz, V. R. (2004). Identification of Key Pinch Forces Required to Complete Functional Tasks. Journal of Rehabilitation Research and Development, 41(2), 215-224. http://dx.doi.org/10.1682/JRRD.2004.02.0215

Smith, A. M., Cadoret, G., \& St-Amour, D. (1997). Scopolamine Increases Prehensile Force During Object Manipulation by Reducing Palmar Sweating and Decreasing Skin Friction. Experimental Brain Research, 114(3), 578-583. http://dx.doi.org/10.1007/PL00005666

Smith, R. O., \& Benge, M. W. (1985). Pinch and Grasp Strength: Standardization of Terminology and Protocol. The American Journal of Occupational Therapy, 39(8), 531-535. http://dx.doi.org/10.5014/ajot.39.8.531

Tanaka, M., \& Numazawa, Y. (2004). Rating and Valuation of Human Haptic Sensation. International Journal of Applied Electromagnetics and Mechanics, 19(1), 573-579

Trew, M., \& Everett, T. (2005). Human Movement: An Introductory Text (5th ed.). London: Churchill Livingstone.

Valero-Cuevas, F. J., Smaby, N., Venkadesan, M., Peterson, M., \& Wright, T. (2003). The Strength-Dexterity Test as a Measure of Dynamic Pinch Performance. Journal of Biomechanics, 36(2), $265-270$. http://dx.doi.org/10.1016/S0021-9290(02)00340-8

Winstein, C. J., Abbs, J. H., \& Petashnick, D. (1991). Influences of Object Weight and Instruction on Grip Force Adjustments. Experimental Brain Research, 87(2), 465-469. http://dx.doi.org/10.1007/BF00231864

Zackrisson, T., Eriksson, B., Hosseini, N., Johnels, B., \& Krogstad, A. L. (2008). Patients with Hyperhidrosis Have Changed Grip Force, Coefficient of Friction and Safety Margin. Acta Neurologica Scandinavica, 117(4), 279-284. http://dx.doi.org/10.1111/j.1600-0404.2007.00938.x

\section{Copyrights}

Copyright for this article is retained by the author(s), with first publication rights granted to the journal.

This is an open-access article distributed under the terms and conditions of the Creative Commons Attribution license (http://creativecommons.org/licenses/by/3.0/). 